\title{
Eficácia biológica de bifentrina aplicado em milho armazenado sob diferentes temperaturas
}

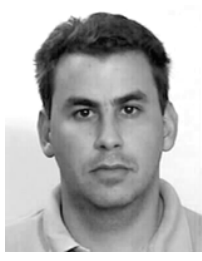

Marco A. G. Pimentel ${ }^{1}$, Lêda R. D'Antonino Faroni² ${ }^{2}$ Raul N. C. Guedes ${ }^{3}$, José R. Gonçalves ${ }^{4} \&$ Carlos R. F. de Oliveira ${ }^{4}$

\author{
DEA/UFV. CEP 36570-000, Viçosa, MG. Fone: (31) 3899-1916. E-mail: marco@insecta.ufv.br (Foto) \\ DEA/UFV. Fone: (31) 3899-1874. E-mail: Ifaroni@ufv.br \\ 3 DBA/UFV. Fone: (31) 3899-2006. E-mail: guedes@ufv.br \\ 4 DBA/UFV. Fone: (31) 3899-1919. E-mail: goncalves_mip@hotmail.com; crfoliveira@hotmail.com
}

Protocolo $116-7 / 7 / 2003$ - Aprovado em 18/10/2004

\begin{abstract}
Resumo: Considerando-se as altas temperaturas nos graneleiros junto à esteira transportadora de grãos objetivou-se, neste trabalho, avaliar a influência da temperatura no momento da pulverização, sobre a eficácia biológica do bifentrina. Para isso, pulverizou-se o inseticida sobre grãos de milho dentro de uma câmara climática nas temperaturas de 25, 30, 35, 40, 45 e 50 ${ }^{\circ} \mathrm{C}$, com umidade relativa em torno de $55 \%$. Após a pulverização e a cada 15 dias, até completar 90 dias, foram feitas as análises da eficácia biológica utilizando-se os insetos Sitophilus zeamais Motschulsky (Coleoptera: Curculionidae), Rhyzopertha dominica (Fabricius) (Coleoptera: Bostrichidae) e Tribolium castaneum (Herbst) (Coleoptera: Tenebrionidae). Observou-se tendência decrescente da eficácia biológica do bifentrina com o aumento da temperatura do ar ambiente, no momento da pulverização e com o maior tempo de armazenamento dos grãos de milho, resultando em menor mortalidade dos insetos-praga.
\end{abstract}

Palavras-chave: pragas de armazenamento, controle químico, bifentrina

\section{Biological efficacy of applied bifenthrin in stored corn under different temperatures}

\begin{abstract}
Considering the high temperatures in the granary ships alongwith the transporting mat, the objective of this paper was to evaluate the influence of the temperature at the moment of spraying on the biological effectiveness of the bifenthrin. For the purpose the insecticide was sprayed on maize grains inside a climatic chamber maintained at the temperatures of 25, 30, 35, 40,45 and $50{ }^{\circ} \mathrm{C}$ with relative humidity around $55 \%$. After the spraying and every fifteen days up to 90 days, analyses of the biological effectiveness were made by using insects of the Sitophilus zeamais Motschulsky (Coleoptera: Curculionidae), Rhyzopertha dominica (Fabricius) (Coleoptera: Bostrichidae) and Tribolium castaneum (Herbst) (Coleoptera: Tenebrionidae). A decreasing tendency of the biological effectiveness of the bifenthrin was observed with the increase of the air temperature at the moment of spraying and with the increased time of maize storage, resulting in a smaller mortality of the insect-pest.
\end{abstract}

Key words: storage pests, chemical control, bifenthrin

\section{INTRODUÇÃO}

O uso de inseticidas na proteção de grãos armazenados tem grande importância para as unidades armazenadoras (Evans, 1985; White \& Leesch, 1996). No entanto, existem poucos produtos registrados para o controle de insetos-praga, pois deles são exigidos uma série de vantagens e padrões de segurança (Watters et al., 1983; Lethbridge, 1989; Hagstrum \& Flinn, 1996). Quanto às vantagens, esses produtos devem persistir por longos períodos em concentrações letais para as pragas, ser de aplicação mais segura que os fumigantes e não requerer equipamentos especiais ou metodologia sofisticada. Com relação aos padrões de segurança, os inseticidas aprovados precisam ser seletivos, apresentar baixa toxicidade para mamíferos e não deixar resíduos tóxicos acima do limite prescrito pelas fontes legais, além de não reduzirem a viabilidade das sementes (Harein \& Las Casas, 1974).

$\mathrm{Na}$ maioria das vezes, a eficiência dos inseticidas fica comprometida com a degradação e com o mau uso desses 
produtos. Muitos são os fatores que podem afetar o processo de degradação dos inseticidas, sendo que o aumento da temperatura e do teor de umidade do grão faz com que a degradação do clorpirifós metílico seja mais rápida (Desmanchelier \& Bengston, 1979; Snelson, 1987).

Pesquisas sobre a degradação de inseticidas e sua eficácia biológica vêm sendo desenvolvidas com sucesso havendo, entretanto, carência de estudos sobre o efeito de condições climáticas extremas no momento da aplicação. Levando-se em consideração o fato do Brasil ser um país de clima tropical, a temperatura do ar ambiente próximo à correia transportadora de um graneleiro pode, nas horas mais quentes do dia, superar os $50{ }^{\circ} \mathrm{C}$ (Hamacher et al., 2002). Considerando-se que a temperatura interfere na ação dos inseticidas, desenvolveu-se este trabalho com o propósito de avaliar o efeito da temperatura no momento da pulverização, sobre a degradação dos resíduos e a eficácia biológica do piretróide bifentrina, o qual vem sendo utilizado com freqüência nas unidades armazenadoras de grãos do Brasil.

\section{MATERIAL E MÉTODOS}

O inseticida avaliado foi o bifentrina (ProStore 25CE), que pertence ao grupo químico dos piretróides. A dosagem utilizada foi de $0,4 \mathrm{ppm}$, o que corresponde a $16 \mathrm{~mL}$ do produto comercial para $1,5 \mathrm{~L}$ de água.

O milho utilizado neste trabalho foi o da variedade AG 1051, tipo mole, com unidade de $13 \%$ b.u. Para a pulverização dos grãos utilizou-se um pulverizador, que consiste de um depósito de calda, bomba de pressão constante a gás $\left(\mathrm{CO}_{2}\right)$, manômetro, conjunto de mangueiras, filtros e bico de pulverização que, por sua vez foi regulado para fornecer uma vazão de calda igual a $17,40 \mathrm{~L} \mathrm{~h}^{-1}$, de modo a proporcionar uniformidade de aplicação. O conjunto de pulverização foi instalado em uma correia transportadora com dimensões compatíveis com a câmara climática $\left(8 \mathrm{~m}^{3}\right)$. Sua capacidade de transporte é de $11,5 \mathrm{th}^{-1}$, sendo acionada por um motor de indução trifásico, acoplado a um moto-redutor, com redução de 24 vezes, ambos de $0,5 \mathrm{CV}$. Para alimentação da correia utilizou-se uma moega com regulagem de vazão permitindo-se, assim, um fluxo uniforme de grãos em toda a extensão da correia transportadora.

Foram utilizados, neste estudo, insetos adultos provenientes de criação massal do Laboratório de MIP-Grãos da Universidade Federal de Viçosa, em Viçosa, MG. As espécies utilizadas foram Sitophilus zeamais Motschulsky (Coleoptera: Curculionidae), Rhyzopertha dominica (Fabricius) (Coleoptera: Bostrichidae) e Tribolium castaneum (Herbst) (Coleoptera: Tenebrionidae). Esses coleópteros foram criados em frascos de vidro com cerca de $1,5 \mathrm{~kg}$ de milho, com $13 \%$ b.u., em câmara climática do tipo B.O.D., na temperatura de $30 \pm 1{ }^{\circ} \mathrm{C}$, umidade relativa de $65 \pm 5 \%$ e escotofase de $24 \mathrm{~h}$. Por se tratar de uma praga secundária, o T. castaneum foi mantido em grãos de milho semitriturados.

Para avaliação da eficácia biológica do bifentrina em grãos de milho, simulou-se, em câmara climática, o efeito de seis temperaturas do ar ambiente: $25,30,35,40,45$ e $50^{\circ} \mathrm{C}$ e uma umidade relativa constante de $55 \%$, no momento da pulverização dos grãos. Em seguida à pulverização, os grãos tratados foram homogeneizados e acondicionados em sacos de polietileno, cujas amostras foram armazenadas em câmara climática tipo B.O.D., a $27{ }^{\circ} \mathrm{C}$ e UR em torno de $60 \%$, até o momento de realização dos ensaios. A seguir e a cada 15 dias, até 90 dias da pulverização, 20 insetos adultos, não-sexados, de cada espécie em estudo, foram inoculados, separadamente, em três placas de Petri $(140 \mathrm{~mm}$ de diâmetro por $10 \mathrm{~mm}$ de altura) contendo $20 \mathrm{~g}$ de grãos de milho tratados com o inseticida.

Após a infestação, as placas foram colocadas em câmaras climáticas tipo B.O.D., nas mesmas condições em que se manteve a criação dos insetos-praga. As avaliações foram realizadas 24 e 48 horas após a infestação, contando-se o número de insetos mortos. Os dados obtidos nessas contagens foram correlacionados com a testemunha, a qual foi pulverizada apenas com água destilada, nas mesmas condições de temperatura e umidade relativa do ar, no momento da pulverização, por meio da fórmula Abbot (1925): $\mathrm{Ef}=(\mathrm{Mtr}-$ Mte)/(100-Mte); onde: $\mathrm{Ef}=$ eficácia do tratamento (\%); $\mathrm{Mtr}=$ mortalidade no tratamento $(\%) ;$ Mte $=$ mortalidade da testemunha (\%).

Os dados avaliados foram submetidos à análise de covariância e, eventualmente, à análise de regressão (SAS, 1989).

\section{RESULTADOS E DISCUSSÃO}

Foram observadas reduções na mortalidade de $S$. zeamais (de $45 \%$ para valores próximos a $5 \%$ ) após $24 \mathrm{~h}$ de exposição a temperatura de $25^{\circ} \mathrm{C}$, com o período de armazenamento (Figura 1). Resultados semelhantes foram observados por Hamacher et al. (2002) para o pirimifós metílico sobre a mortalidade de $S$. zeamais. A redução da eficácia biológica de inseticidas protetores durante o período de armazenamento de grãos, na mortalidade de espécies de Sitophilus, tem sido relatada por vários pesquisadores. Bitran et al. (1991) utilizando o inseticida deltametrina na dose de $0,5 \mathrm{ppm}$, observaram que após o período de seis meses de armazenamento dos grãos, em temperaturas do ar ambiente variando entre 19 e $26^{\circ} \mathrm{C}$, a eficácia desse inseticida sobre o controle de $S$. zeamais foi reduzida consideravelmente. Daglish (1998), associando deltametrina com clorpirifós-metílico e metacrifós, observou $64 \%$ de mortalidade da progênie de Sitophilus oryzae (L.) após 84 dias de ser pulverizado sobre trigo, nas condições de temperatura e umidade relativa do ar de $30{ }^{\circ} \mathrm{C}$ e $55 \%$, respectivamente. Já Fleurat-Lessard et al. (1998) observaram, em experimentos com clorpirifós-metílico pulverizado a $30^{\circ} \mathrm{C}$ que, a partir de 60 dias de armazenamento, a eficácia do inseticida sobre $S$. oryzae começou a cair consideravelmente, passando de $100 \%$ de eficácia aos 60 dias, para menos de $20 \%$ aos 126 dias de armazenamento.

Para $R$. dominica, observaram-se os menores níveis de eficiência do bifentrina, 24 e 48 h após a aplicação (Figura 2), o que pode estar indicando resistência desse inseto ao piretróide (Gonçalves et al., 2002). Esta resistência vem sendo observada por outros pesquisadores, uma vez que $R$. dominica tem apresentado comportamento semelhante quando são usados outros piretróides como biorresmetrina, fenotrina, fenvalerate 
A.

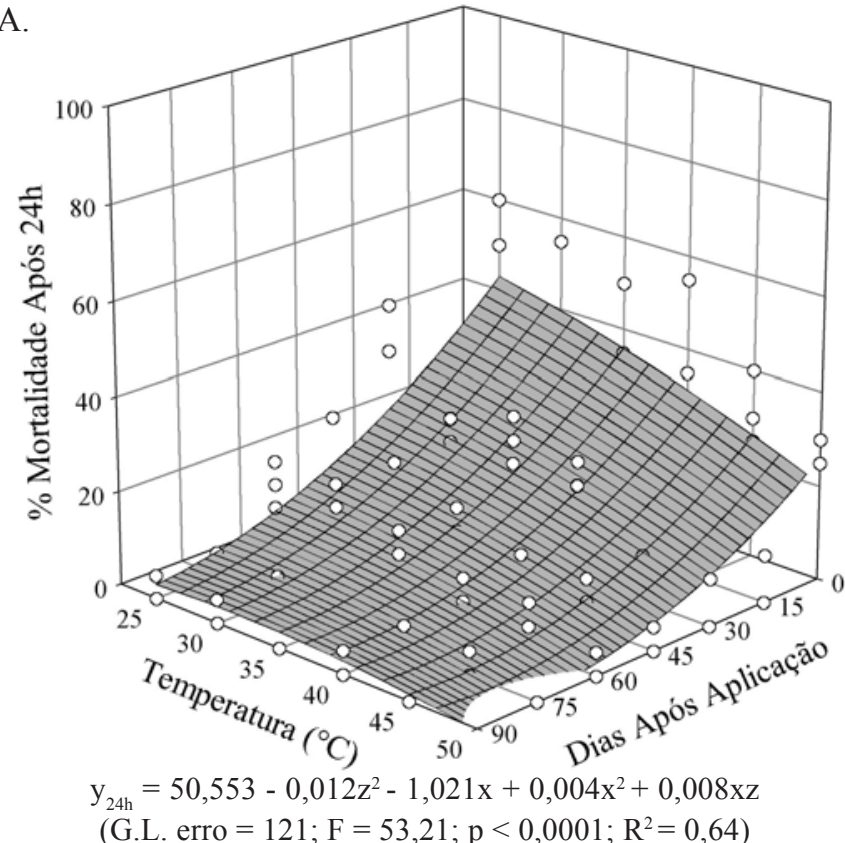

B.

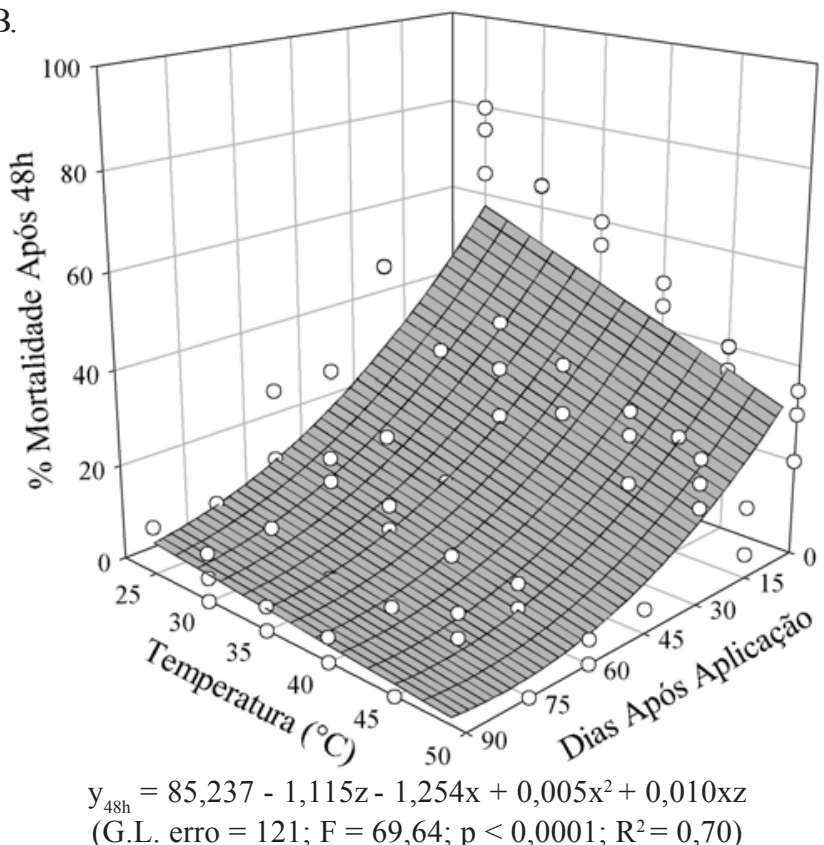

Figura 1. Efeito da temperatura do ar ambiente no momento da pulverização de grãos de milho com bifentrina e do tempo de armazenamento na mortalidade $\mathrm{y}_{\mathrm{hs}}$ de Sitophilus zeamais após 24 (A) e $48 \mathrm{~h}(\mathrm{~B}), \mathrm{x}=$ dias após aplicação e $\mathrm{z}=$ temperatura $\left({ }^{\circ} \mathrm{C}\right)$

A.

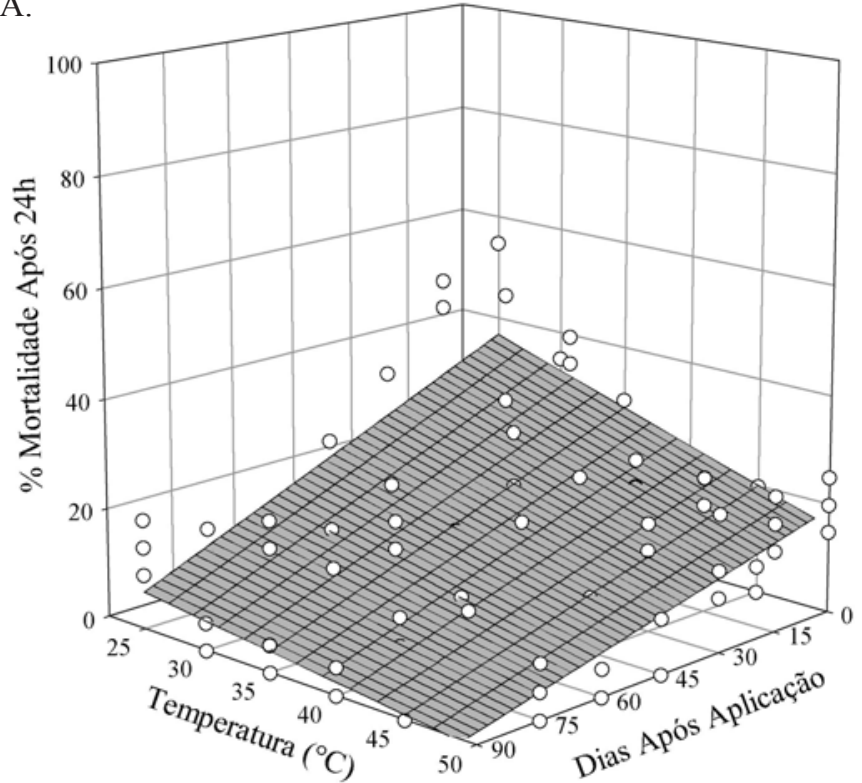

$\mathrm{y}_{24 \mathrm{~h}}=57,629-0,833 \mathrm{z}-0,482 \mathrm{x}+0,006 \mathrm{xz}$

(G.L. erro $\left.=122 ; \mathrm{F}=69,25 ; \mathrm{p}<0,0001 ; \mathrm{R}^{2}=0,63\right)$
B.

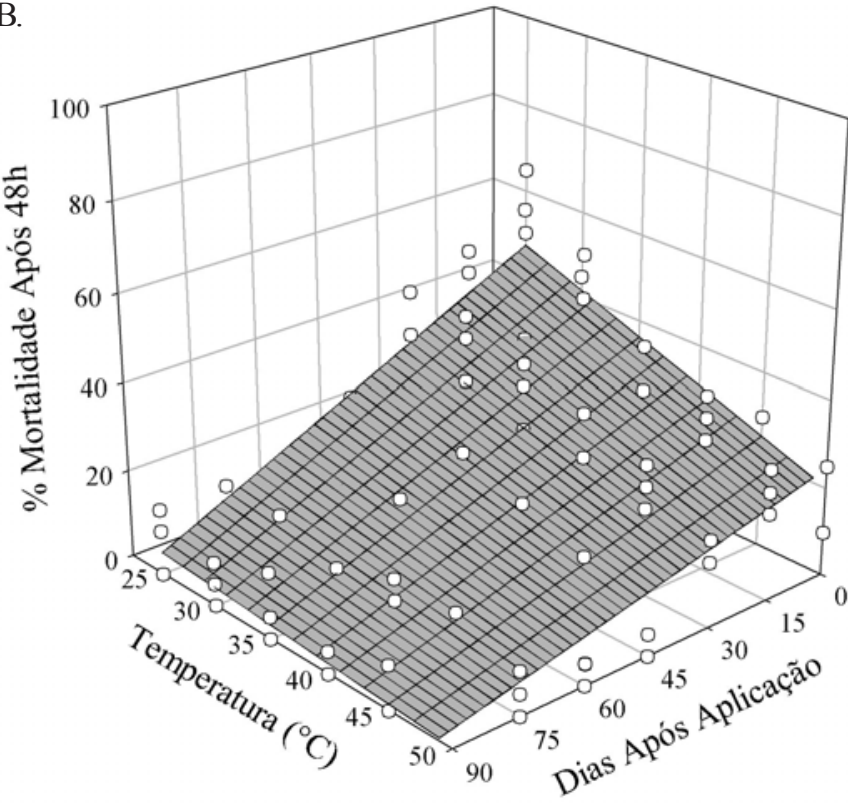

$\mathrm{y}_{48 \mathrm{~h}}=73,833-1,075 \mathrm{z}-0,714 \mathrm{x}+0,010 \mathrm{xz}$

(G.L. erro $\left.=122 ; \mathrm{F}=62,21 ; \mathrm{p}<0,0001 ; \mathrm{R}^{2}=0,60\right)$

Figura 2. Efeito da temperatura do ar ambiente no momento da pulverização de grãos de milho com bifentrina e do tempo de armazenamento na mortalidade $\mathrm{y}_{\mathrm{hs}}$ de Rhyzopertha dominica, após 24 e $48 \mathrm{~h},(\mathrm{~A}), \mathrm{x}=$ dias após aplicação e $\mathrm{z}=$ temperatura $\left({ }^{\circ} \mathrm{C}\right)$

(Collins et al., 1993), deltametrina (Collins et al., 1993; Lorini \& Galley, 1999; 2000) e os organofosforados malation, pirimifós metílico e clorpirifós metílico (Guedes et al., 1996; Guedes \& Zhu, 1998). Outras pragas de grãos armazenados como $S$. oryzae, $S$. zeamais e $T$. castaneum também têm apresentado resistência aos piretróides (Heather, 1986; Collins, 1990; PerezMendoza, 1999). Embora R. dominica mostre-se tolerante ao bifentrina, a maior redução da eficácia desse inseticida foi observada com o aumento da temperatura e do período de armazenamento. Tal redução também foi relatada por Bitran et al. (1991) em experimentos utilizando trigo tratado com deltametrina na dosagem de $0,5 \mathrm{ppm}$, ao longo de seis meses de armazenamento, em temperaturas de $25^{\circ} \mathrm{C} \mathrm{em}$ média.

Os melhores resultados da eficácia biológica do bifentrina foram obtidos após $48 \mathrm{~h}$ de exposição de $T$. castaneum aos resíduos do inseticida; no entanto, a mortalidade desse inseto diminuiu com o aumento do período de armazenamento, reduzindo-se de $75 \%$ para valores próximos a $20 \%$ após $24 \mathrm{~h}$ de 

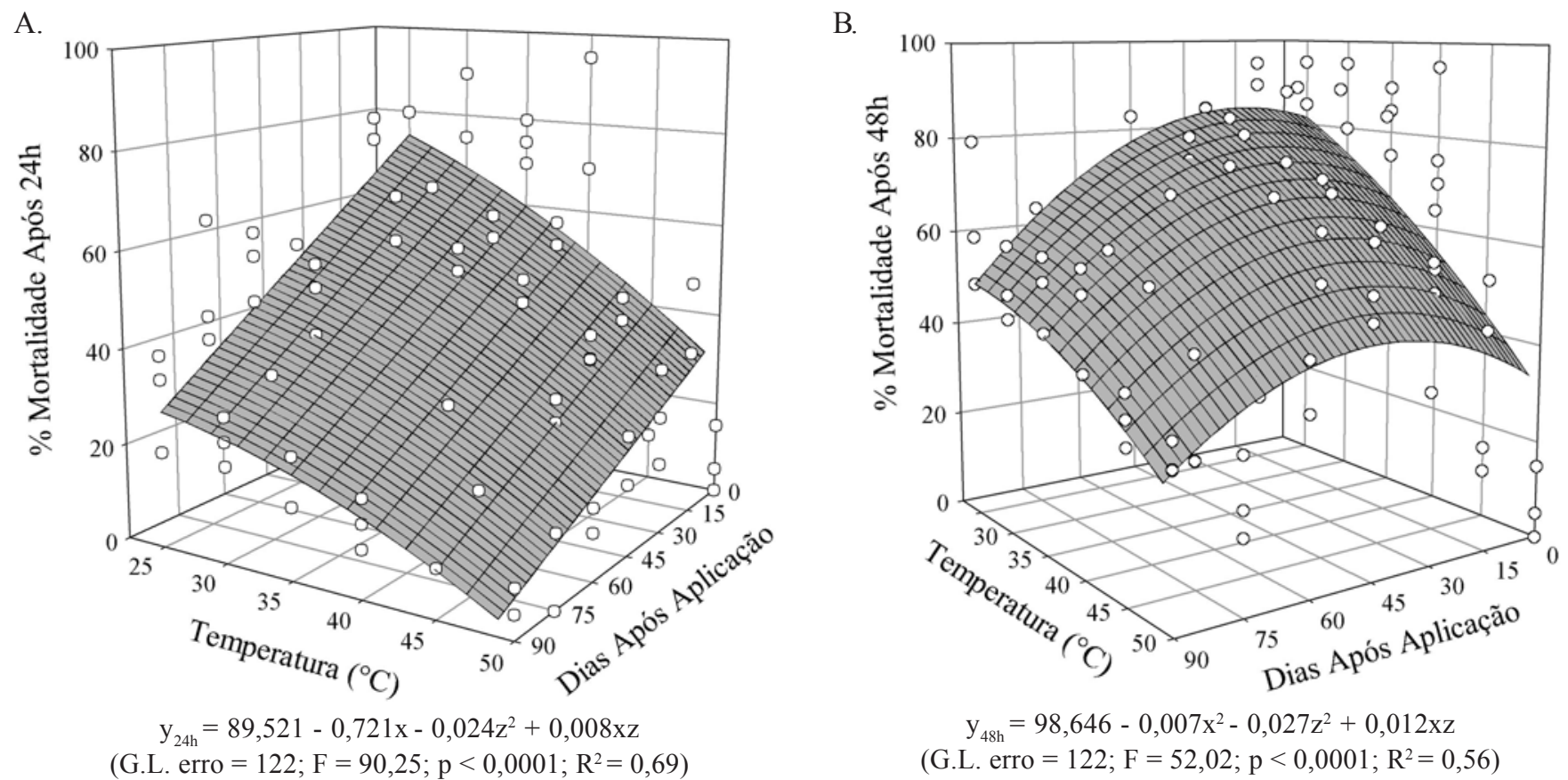

Figura 3. Efeito da temperatura do ar ambiente no momento da pulverização de grãos de milho com bifentrina e do tempo de armazenamento na mortalidade $\mathrm{y}_{\mathrm{hs}}$ de Tribolium castaneum. (A) e após $48 \mathrm{~h}(\mathrm{~B}), \mathrm{x}=$ dias após aplicação e $\mathrm{z}=$ temperatura $\left({ }^{\circ} \mathrm{C}\right)$

exposição, na temperatura de $25^{\circ} \mathrm{C}$ (Figura 3). Resultados semelhantes foram relatados por Fleurat-Lessard et al. (1998) em experimentos com clorpirifós metílico pulverizado na temperatura de $30^{\circ} \mathrm{C}$, quando observaram que, a partir de 42 dias de armazenamento, a eficácia do inseticida começou a declinar, atingindo $0 \%$ de mortalidade de T. castaneum aos 126 dias de armazenamento. Esta tendência também foi constatada por Barson (1983) e Guedes et al. (1992) em experimentos em que relacionavam, simultaneamente, os efeitos de temperatura e umidade relativa do ar ambiente sobre a atividade biológica dos inseticidas.

Em geral, os inseticidas de contato apresentam características que proporcionam longo período de proteção para os grãos armazenados contra insetos-praga (Snelson, 1987). Entretanto, esses produtos podem apresentar perdas de sua eficácia de acordo com a maneira como são pulverizados sobre os grãos (Johnson, 1990; Arthur et al., 1991; Hamacher et al., 2002).

O estudo da eficácia biológica de piretróides em altas temperaturas é de suma importância, pelo fato desses inseticidas serem pulverizados com freqüência em regiões tropicais, nas horas mais quentes do dia, o que favorece a degradação do produto e menor proteção dos grãos. Uma solução simples para minimizar este efeito seria pulverizar os inseticidas protetores de grãos nos períodos em que as temperaturas sejam mais brandas ou durante a noite.

\section{CONCLUSÃO}

A eficácia biológica do inseticida bifentrina sobre a mortalidade dos insetos-praga $R$. dominica, $S$. zeamais e $T$. castaenum, é reduzida com o aumento da temperatura do ar ambiente, no momento da pulverização e ao longo do período de armazenamento.

\section{LITERATURA CITADA}

Abbott, W.S. A method of computing the effectiveness of an insecticide. Journal of Economic Entomology, Lanham, v.18, n.1, p.265-267, 1925.

Arthur, F.H.; Throne, J.E; Simonaitis, R.A. Chlorpyripos-methyl degradation and biological efficacy toward maize weevils (Coleoptera: Curculionidae) on corn stored at four temperatures and three moisture contents. Journal of Economic Entomology, Lanham, v.84, n.1, p.1926-1932, 1991.

Barson, G. The effects of temperature and humidity on the toxicity of three organophosphorus insecticides to adult Oryzaephilus surinamensis (L.). Pesticide Science, London, v.14, n.1, p.145-152, 1983.

Bitran, E.A.; Campos, T.B.; Suplicy Filho, N.; Chiba, S. Avaliação da ação residual de alguns inseticidas na proteção de grãos de milho, trigo e arroz contra pragas de armazenamento. Arquivos do Instituto Biológico de São Paulo, São Paulo, v.58, n.1/2, p.43-50, 1991.

Collins, P.J. New resistance to pyrethroids in Tribolium castaneum (Herbst.). Pesticide Science, London, v.28, n.1, p.101-115, 1990 .

Collins, P.J.; Lambkin, T.M.; Bridgeman, B.W.; Pulvirenti, C. Resistance to grain-protectant insecticides in coleopterons pests of stored cereals in Queensland, Australia. Journal of Economic Entomology. Lanham, v.86, n.1, p.239-245, 1993.

Daglish, G.J. Efficacy of six grain protectants applied alone or in combination against three species of coleoptera. Journal of Stored Products Research, Oxford, v.34, n.4, p.263-268, 1998. 
Desmanchelier, J.M.; Bengston, M. The residual behavior of chemicals on stored grain. In: International Working Conference on Stored-Product Entomology, 2, 1979, Ibadan, Nigeria. Proceedings... Ibadan, 1979, p.138-151.

Evans, N.J. The effectiveness of various insecticides on some resistant beetle pest of stored products from Uganda. Journal of Stored Products Research, Oxford, v.21, n.2, p.105109,1985

Fleurat-Lessard, F.; Vidal, M.L.; Budzinski, H. Modeling biological efficacy decrease and rate of degradation of chlorpyrifos-methyl on wheat stored under controlled conditions. Journal of Stored Products Research, Oxford, v.34, n.4, p.341-354, 1998.

Gonçalves, J.R.; Faroni, L.R.D’A.; Guedes, R.N.C. PyrethoidAcarophenax lacunatus interaction in suppressing the beetle Rhyzopertha dominica on stored wheat. Experimental and Applied Acarology, Amsterdam, v.26, n.1, p.231-242, 2002.

Guedes, R.N.C.; Dover, B.A.; Kambhampati, S. Resistance to chlorpyrifos-methyl, pirimiphos-methyl, and malathion in Brazilian and U.S. populations of Rhyzopertha dominica (Coleoptera: Bostrichidae). Journal of Stored Products Research, Oxford, v.89, n.1, p. 27-32, 1996.

Guedes, R.N.C.; Heyde, C.J.; Silva, F.A.P.; Picanço, M.C. Efeito da temperatura e da umidade relativa do ar na toxicidade de bifentrina a Sitophilus zeamais (Coleoptera: Curculionidae). Revista Brasileira de Armazenamento, Viçosa, v.17, n.1, p.4448, 1992.

Guedes, R.N.C.; Zhu, K.Y. Characterization of malathion resistance in a Mexican population of Rhyzopertha dominica. Pesticide Science. London, v.53, n.1, p.15-20, 1998.

Hagstrum, D.W.; Flinn, P.W. Integrated pest management. In: Subramanyam, BH.; Hagstrum, D.W. (eds.). Integrated Management of Insects in Stored Products. New York: Marcel Dekker, 1996. cap. 9, p.399-408.

Hamacher, L.S.; Faroni, L.R.D’A.; Guedes, R.N.C.; Queiróz, M.E.L.R. Persistence and activity towards Sitophilus zeamais (Coleoptera: Curculionidae) of pirimiphos-methyl sprayed at different temperatures on maize. Journal of Stored Products Research, Oxford, v.38, n.2, p.167-175, 2002.
Harein, P.H.; Las Casas, E. Chemical control of stored-grain insect and associated micro and macro organisms. St. Paul: Association of Cereal Chemistry, p.232-291, 1974.

Heather, N.W. Sex-linked resistance to pyrethroids in Sitophilus oryzae (L.) (Coleoptera: Curculionidae). Journal of Stored Products Research, Oxford, v.22, n.1, p.15-20, 1986.

Johnson, D.L. Influence of temperature on toxicity of two pyrethroids to grasshoppers (Orthoptera: Acrididae). Journal of Economic Entomology, Lanham, v.83, n.1, p.366-373, 1990.

Lethbridge, G. An industrial view of microbial inoculants for crop plants. In: Campbell, R.; MacDonald, R.M. (eds.) Microbial inoculation of crop plants. Oxford: Pergamon Press, 1989. p.11-28.

Lorini, I.; Galley, D.J. Deltamethrin resistance in Rhyzopertha dominica (F.) (Coleoptera: Bostrichidae), a pest of stored grain in Brazil. Journal of Stored Products Research, Oxford, v.35, n.1, p.37-45, 1999.

Lorini, I.; Galley, D.J. Estimation of realized heritability of resistance to deltamethrin insecticide in selected strains of Rhyzopertha dominica (F.) (Coleoptera: Bostrichidae). Journal of Stored Products Research, Oxford, v.36, n.1, p.119124, 2000.

Perez-Mendoza, J. Survey of insecticide resistance in Mexican populations of maize weevil, Sitophilus zeamais Motschulsky (Coleoptera: Curculionidae). Journal of Stored Products Research, Oxford, v.35, n.1, p.107-115, 1999.

SAS. SAS/STAT User's guide, version 6.Cary: SAS Institute, 1989, v.6, p.1256-1294.

Snelson, J.T. Grain protectants. Camberra, Australian Center for International Research, 1987, 448p. Monograph, 3

Watters, F.L.; White, N.D.G.; Coté, D. Effect of temperature on the toxicity and persistence of three pyrethroid insecticides applied to fir plywood for the control of Tribolium castaneum. Journal of Economic Entomology, Lanham, v.76, n.1, p.11-16, 1983.

White, N.D.G.; Leesch, J.G. Chemical control. In: Subramanyam, BH.; Hagstrum, D.W. (eds.) Integrated management of insects in stored products. New York: Marcel Dekker, 1996, cap.7. p.287-330. 